\title{
Cross-Topic Author Identification - a Case Study on Swedish Literature
}

\author{
Niklas Zechner \\ Språkbanken \\ Department of Swedish \\ University of Gothenburg \\ niklas.zechner@gu.se
}

\begin{abstract}
Using material from the Swedish Literature Bank, we investigate whether common methods of author identification using word frequencies and part of speech frequencies are sensitive to differences in topic. The results show that this is the case, thereby casting doubt on much previous work in author identification. This sets the stage for a broader future study, comparing other methods and generalising the results.
\end{abstract}

\section{Introduction}

Author identification is a competitive field, with many studies reporting ever increasing accuracies. Often, the accuracy as reported by the experiment is seen as irrefutable proof that the method works. But there may be reason to be sceptical of the optimistic results. Previous work has shown that there are several things to take into account for text classification generally, before methods can be considered reliable and comparable. The size of the texts has a large impact on the accuracy, and naturally the number of candidate classes also matters (Zechner, 2017). Even minor details in how the test data is handled can lead to significant overestimation of the accuracy (Zechner, 2014).

When it comes to author identification specifically, one of the main pitfalls is neglecting to account for differences in topic, style, or genre (Mikros and Argiri, 2007). If we apply a classification method to texts by several different authors, but each author mainly writes on a particular topic, how do we know if the classification method is detecting authors or topics? If the method is sensitive to topic, the accuracy reported in testing may be far higher than what we would get from a real-life application, where the text to be identified is on a different topic. Ideally, we would like to test this using texts marked for both topic and author, but performing such a study would be difficult at best - not only would it be hard to find a large corpus marked for topic, it is also doubtful if any two texts can be said to be on exactly the same topic.

The question of what topic really means is of course a matter of both debate and opinion, but that discussion is not really relevant here. For our purposes, we can essentially define topic as everything that is not author - any traits of a text which do not correspond to traits of the author can be considered effects of the "topic", including genre, medium, level of formality, and so on.

Many have tried to get around the problem by basing their methods on features of the text which are assumed to be independent of topic. Perhaps the most famous example is by Mosteller and Wallace (1964), in their study on the Federalist Papers. They based their analysis on the frequencies of function words, that is, words whose meaning is mainly grammatical rather than semantic, arguing that those words should not be dependent on topic. But they did not put that assumption to the test, and few have done since. While it may seem sensible to think that simple grammatical words like "the" or "of" should be used with about the same frequency across all topics, it is arguably just as sensible to say that they should be used equally by all authors.

Since it is unfeasible to find texts on the same topic by different authors, we have to approach the problem differently. One thing we can find is texts by the same author that can be considered different in topic, at least in this broad sense. Using a corpus of such texts, we can compare how well a method performs in different situations - is the accuracy lower when the texts we try to match up are on different topics? We can also apply the same method to identifying a topic among texts by the same author, which gives us another indication of how sensitive the method is to topic.

In a previous study (Björklund and Zechner, 
2017), we investigated this problem by examining a set of novels, using each separate novel as an approximation of topic. In this study, we begin to expand on that work and apply a similar approach to a larger corpus, this time in Swedish.

As an alternative to function words, some have tried using features based on grammatical analysis of the words. Could the grammatical patterns of an author be less topic-dependent than their use of function words? Different studies have given conflicting results, finding such methods to be worse (Menon and Choi, 2011), equally good (Luyckx and Daelemans, 2005), or better (Björklund and Zechner, 2017). We apply a method using parts of speech alongside the word-based method to see if there are differences in how they relate to topics.

\subsection{The problem}

In a typical author identification task, we want to find which of a set of candidate texts is written by the same author as a given target text. To test a method on this task, we need a number of text samples, at least two of which are by the same author. One of the two acts as the target text, and one is mixed in with texts by other authors to form the candidates. We now have a set of candidate texts with one "true" candidate, the one which is actually by the same author as the target text, and some number of "false" candidates, which are by other authors. If the method correctly identifies the true candidate, it is considered successful. By repeating the experiment, we can estimate the accuracy of the method, that is, the probability of successful identification.

Commonly, when we test a method like this, we only have access to an unstructured text or set of texts by each of a number of authors. This could be articles or letters, or internet data such as forum messages or blog texts. This causes a problem when evaluating the test results. If the methods can reliably identify text samples from the same source, is that because they are written by the same author, or is it because they are on a similar topic? There is a risk that the methods look very accurate in a test setting, but are actually much less so when we apply them to a real-life problem.

\subsection{The approach}

To address this issue, we use text samples from books, under the hypothesis that each book can be seen as a separate topic. (Note, again, that we are using "topic" effectively as shorthand for "any systematic difference that is not directly due to the author" - genre, context etc.) This allows us to try three variants of the identification task, as illustrated in Figure 1.

In the first case, the true candidate comes from the same book as the reference text, and the false candidates come from books by other authors. This corresponds to the commonly seen experiment, where we are effectively identifying a combination of author and topic. In the second case, the true candidate is from the same author as the reference text, but not from the same book, and the false candidates are again texts from other authors. This way, we are identifying author without the influence of topic. In the third case, the true candidate is again from the same book as the reference text, but the false candidates are now from other books by the same author. Now we are identifying only topic, without the influence from author. By comparing the results, we hope to see if the method is more sensitive to author or topic.

Using books also has the advantage that we get a large amount of text for each author and topic, which helps reach a reasonable accuracy with simple methods. We will not attempt to make the method as accurate as possible, but rather keep it simple and transparent. This is because the goal here is not promoting a method, but rather showing the effects of topic on existing methods.

\section{Data}

We use data taken from the Swedish Literature Bank (litteraturbanken.se), a collection of old novels, from which we include only the ones that have been manually digitised. We restrict the data to works in Swedish, by a single known author, and leave out works that contain duplicate text, such as multiple editions of the same book. This leaves 481 books by 140 authors.

Each book is cut up into pieces of 40000 words, leaving out any trailing words. One reason for this is so that the texts are all the same lengths, making the results meaningful and reproducible. Previous work has found that the accuracy of classification varies greatly with the length of texts, so that if we were to include entire books of varying length, the experiments would have little predictive value (Zechner, 2017). Another reason is that we want to compare texts from the same book, so it is necessary to divide at least some of the books into parts. We get 825 pieces in total. 

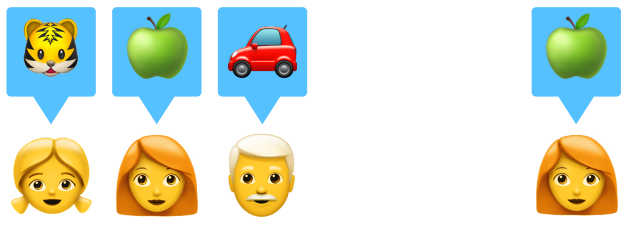

Case one: Identifying a text based on both topic and author. The correct candidate sample is from the same book as the target sample. The other candidate samples are by other authors.
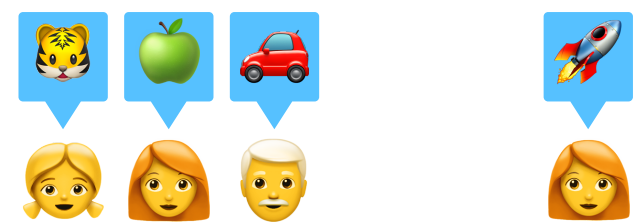

Case two: Identifying a text based on only author. The correct candidate sample is from the same author as the target sample, but a different book. The other candidate samples are by other authors.
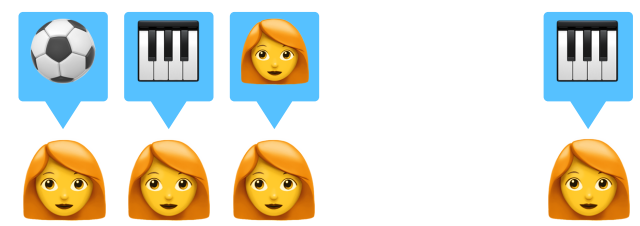

Case three: Identifying a text based on only topic. The correct candidate sample is from the same book as the target sample. The other candidate samples are from other books by the same author.

Figure 1: Illustration of the method.

\section{Method}

We use a feature set consisting of just ten (relative) word frequencies, specifically those words that are the most common in the data generally. "Words" here also include punctuation, and are counted independent of capitalisation. The words in this case are: comma, full stop, "och", "i”, "att”, "det", “en”, "som", "han", "jag".

For each text (that is, for each piece of 40000 words), we create a profile of its frequencies for these ten words. As a distance measure, we calculate the (absolute) difference in each feature value, and sum over all features; in vector terms, this is the Manhattan distance, without any normalisation. Using these profiles, it is easy to compare any pair of text and calculate the distance. That can then be applied to the identification problem as described above, by comparing the target text to each of the candidate texts, and choosing the one with the smallest distance measure.

\subsection{Measuring accuracy}

Now we can run the three tests we want to compare: identifying a book among a set of books by other authors, identifying a book among a set of books by the same author, and identifying an author among others by comparing with a different book by that author. By repeating the process, we can find an estimated accuracy for each case.

But it is possible to go a step further. We can think of each of the possible pairs of texts as being of one of three types: Same book, same author (but different book), and different author. From the 825 chunks analysed, we get in total 537 samebook pairs, 16356 same-author pairs, and 323007 different-author pairs. Since the method is simple and fast, we can easily go though all the possible pairs, and find the distribution of distance measures for each type of pair.

Knowing this distribution has great value in a practical application, because it allows us to calculate the probability that a pairing is of a particular type, and thus the probability that two texts are by the same author, or from the same book. But we can also use it to get a better estimate on the accuracy of the identification problem.

Suppose we want to identify the author of a given text out of 100 candidates, using one other text by that same author and 99 texts by other (not necessarily distinct) authors. This will mean one same-author comparison, and 99 different-author 
comparisons. Using the simplifying assumption that the similarity between a given text and a random text by the same author does not correlate with the average similarity between that given text and a random text by a different author, we do not need to investigate specific text samples one by one. Instead, we can think of it as a simpler statistical problem: For a given same-author pair, how likely is it that it will have a lower distance measure than each of a set of 99 different-author pairs?

To find out, we do not need to choose 99 random different-author pairs. Instead, we keep a sorted list of the different-author pairs. Choosing one same-author pair, we can use a simple binary search to see what fraction $f$ of the different-author pairs have a higher distance measure. Then, the probability of 99 of them having a higher distance measure is just $f^{99}$; this is the probability of this same-author pair being correctly identified. This is simple enough that we can repeat it for all the same-author pairs, and calculate the average accuracy, without having used any random subset.

\subsection{Further variations}

If we look closer at this corpus, we find that there is one author who is far more prolific than the others: August Strindberg. Our sample contains no less than 64 of his works, far more than any other author. Since the number of same-author pairs for an author increases approximately as the square of the number of works by that author, that means that he has a very large impact on the results - about three quarters of the same-author pairs are from Strindberg. This might skew the results, so we run the tests twice, with and without Strindberg.

This corpus also includes a grammatical analysis, so we can try using that as an alternative to word frequencies. In a similar manner, we now count the frequencies of the ten most common parts of speech (POS) (including, again, punctuation).

\section{Results}

The distributions of distance values for the three types of pairs are shown in Figure 2. We can see that the distance values for same-author pairs are lower than those for different-author pairs, as can be expected, but also that the values for same-book pairs are lower still. This immediately tells us that methods like this one would be highly topicdependent. In this graph, the separation between the same-book curve and the same-author curve tells us how strongly the method reacts to topic, and the separation between the same-author curve and the different-author curve tells us how strongly it reacts to author. A small overlap between the same-author curve and the different-author curve would indicate a method which is good for author identification, whereas a small overlap between the same-book curve and the different-author curve would indicate a method which seems good if measured by traditional testing.

The same-book and same-author distributions for Strindberg have been separated out. We can see that they have much higher distance measures, meaning that his works would be much more difficult to identify. Evidently, Strindberg has a more diverse writing style than most; further speculation is beyond the scope of this study.

Figure 3 shows the results of applying the POS method. We see that the results are very similar. The different-author curve still overlaps considerably more with the same-author curve than with the same-book curve, in approximately the same proportions as in Figure 2.

Note that the axes are largely arbitrary; the POS method has higher distance values, because the most common parts of speech have higher frequencies than the most common words, and the y axis is adjusted accordingly due to normalisation. The difference in height and width of the curves between the figures is therefore irrelevant. Also note that while we can see slightly larger overlaps both ways in Figure 3, indicating a lower accuracy, that is also mostly beside the point, since we are not interested in maximising the accuracy.

As outlined in the previous section, we can use the distributions to calculate what would be the average accuracy of an identification test. We choose an identification task with 100 candidates, and try the three different cases: Identifying a book among books by other authors (identification based on both author and topic), identifying an author among others while using a different book as reference (only author), and identifying a book among other books by the same author (only topic). The resulting accuracies are shown in Table 1. We see that in the second case, when we remove the influence of topic, the result is considerably lower, which confirms that the method is not topic-independent. The third case is also on a similar level, suggesting that the sensitivity to topic is in some sense comparable to the sensitivity to author. 


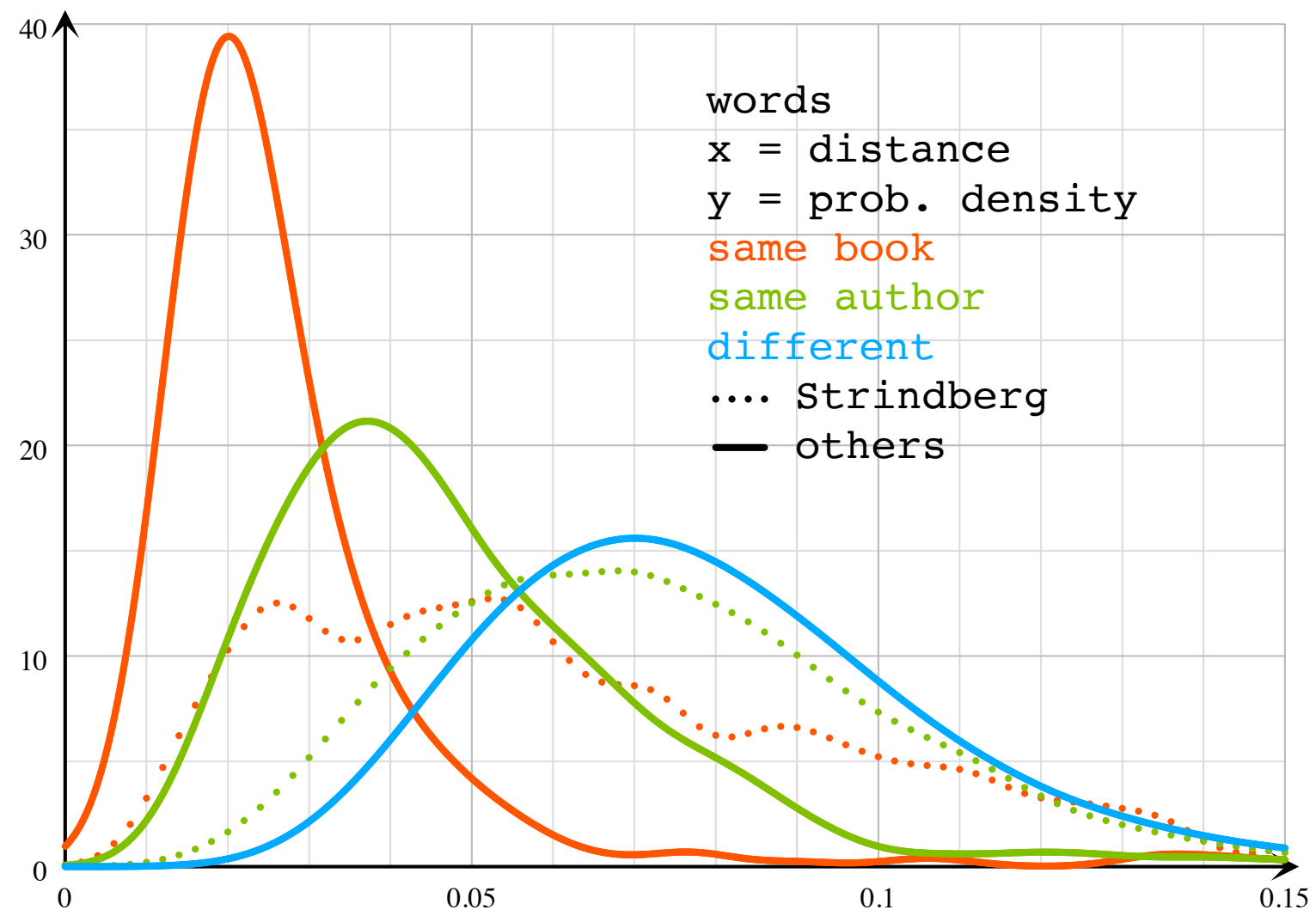

Figure 2: Distributions of distance measures for types of pairs. Distributions sum to one, and have been smoothed with a Gaussian blur, sd $=0.005$. The different-author curve also includes Strindberg.

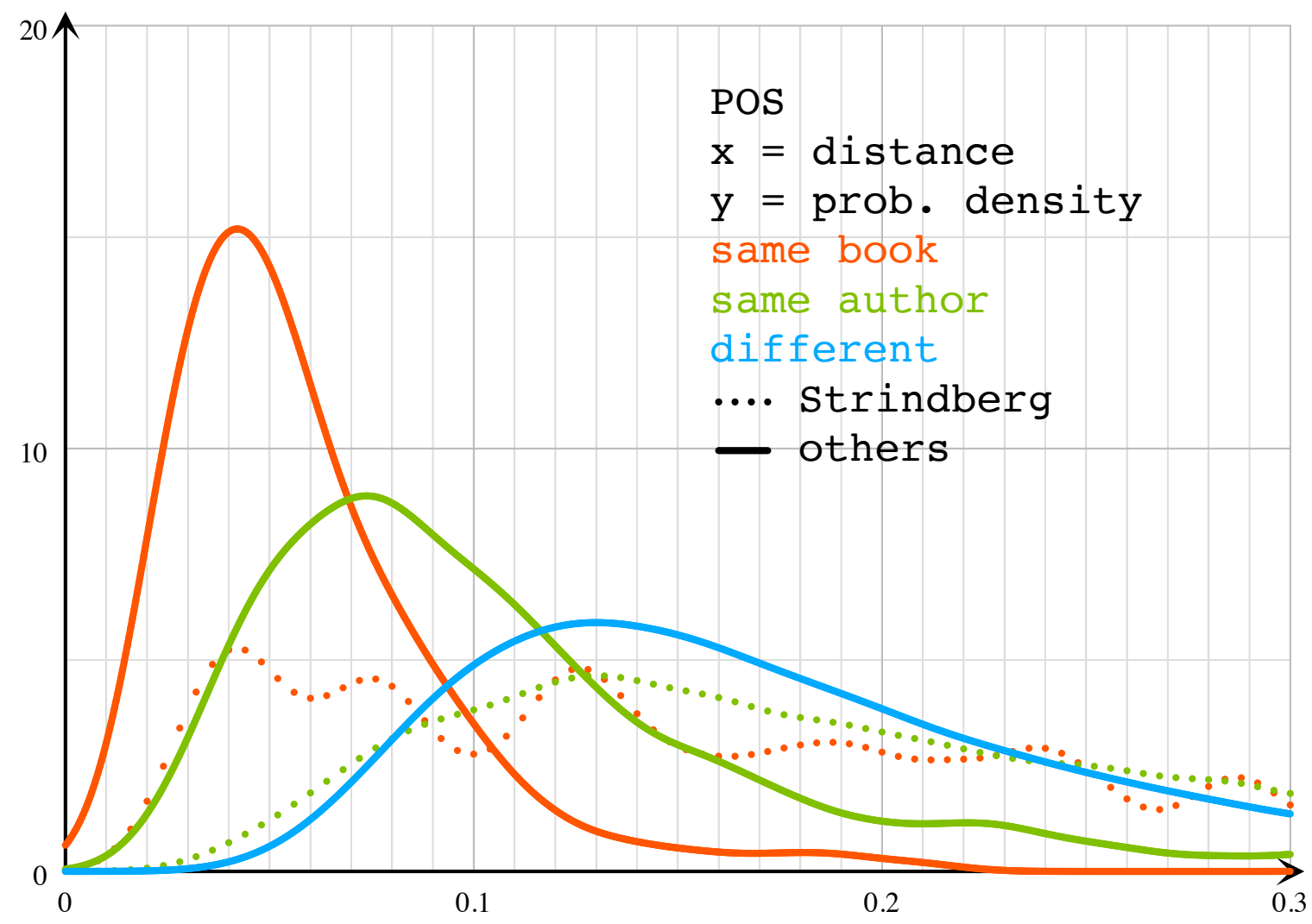

Figure 3: Distributions for POS features. Gaussian blur sd $=0.01$. 


\begin{tabular}{lrl}
\hline Comparison & Words & POS \\
\hline All authors & & \\
same book vs. different & $52 \%$ & $43 \%$ \\
same author vs. different & $8 \%$ & $6 \%$ \\
same book vs. same author & $17 \%$ & $14 \%$ \\
Without Strindberg & & \\
same book vs. different & $67 \%$ & $53 \%$ \\
same author vs. different & $20 \%$ & $17 \%$ \\
same book vs. same author & $11 \%$ & $7 \%$ \\
\hline
\end{tabular}

Table 1: Simulated accuracies for the different tests, for 100 candidates.

The distributions can also be used to calculate the probability that a pair is of a given type. For example, suppose we know that a text sample is either from book A, book B or book C. The three books are by different authors (neither of whom is Strindberg) and we have another sample of book A, but not of book B or C. We compare the unknown sample and the one from book $\mathrm{A}$, and get a distance measure of 0.04 (using the word-based method). How likely is it that the unknown sample is from book A? Since there are three candidates, and we have no further information, the a priori probability is $1 / 3$, or in other words, the a priori probability of a different-author pair is twice as high as that of a same-book pair. Looking at Figure 2, we see that at 0.04 , the same-book curve is at 9 , and the different-author curve at 6 . The final probability for a same-book pair (and therefore, the probability that the unknown sample is from book A) is $1 * 9 /(1 * 9+2 * 6)=43 \%$.

\section{Discussion}

We can see directly from the distribution curves that this method is not topic-independent. The accuracy calculations verify this, and indicate that the method may be at least as sensitive to topic as it is to author. This means that similar methods may not be reliable for author identification; even if experiments show promising results, the accuracy in a real-world application might be far lower.

We should keep in mind that this is not meant as a tool for topic identification; clearly there are far better methods for that. Whether this is an accurate representation of topic is also irrelevant, since we are interested in separating out any traits not related to the author. Furthermore, authors may well write several books on the same topic. But that would only mean that we have underestimated the problem. If we have only partially separated topic from author - as is almost certainly the case - the decrease in accuracy for a real application would be even greater. Future studies may be able to test this using data from more diverse sources.

It should be noted that the methods used here are not intended to be as accurate as possible. We could very likely improve the accuracy by using a larger set of features, or by using some form of normalisation on the feature values, or by using a more advanced classifier. It is also clear from tests not shown here that the accuracy depends heavily on the size of the samples; samples significantly smaller than these would drastically lower the accuracies, and larger samples would improve them. For the same reason, the overall difference in accuracy between the two methods also does not matter.

\subsection{Comparison of methods}

The difference between the analyses based on word features and POS features seems negligible, so these experiments did not reproduce the findings of our previous study on English novels (Björklund and Zechner, 2017). Looking at the results without Strindberg, the gap in accuracy between on the one hand the classic test (the first case in Table 1) and on the other hand the topic-controlled test (the second case) is $70 \%$ for words and $68 \%$ for POS technically a better result for the POS method, but hardly compelling evidence of a difference.

Could a different set of features do better? The words used in the first methods were not chosen specifically to be function words, but it is clear that they are, just as most other common words. Clearly, using function words was not enough to ensure topic independence.

These words have no obvious relation to specific topics, and so there is no obvious way to choose less topic-dependent words. We also know that the amount of data used is a very important factor for accuracy, so unless the texts in question are extraordinarily large, choosing features other than the most common ones would lead to a significant drop in accuracy. Other common features used are word or character n-grams, that is, sequences of several words or characters. It seems quite clear that those would suffer from the same problems.

Different studies have also used many different classifier algorithms. While some would likely give higher accuracies than the simple one used here, we cannot reasonably expect that any other 
standard statistical measure or machine learning algorithm would be less topic-dependent when based on the same topic-dependent features. By using more opaque classifiers like those based on "deep learning", or more opaque feature sets such as character n-grams, we also risk losing the ability to see what the classification choices are based on, which makes it harder to understand problems like that of topic dependence.

\subsection{Future work}

We hope to build on this small experiment towards a larger study of classification on this type of corpus. The large amount of data and clear metadata may be useful for other types of classification, including gender and year of writing. A more comprehensive study of different feature sets might also reveal which types of features are best for identifying authors, which are better for topic, and which are better for identifying something else entirely.

For a future method to be topic-independent, it would likely have to more explicitly address the issue, and separate topic features from author features. This is not in principle impossible; even in writing it is often possible to detect differences in dialect, age of the author, and other personal characteristics which will be stable across topics. Can we automatically detect which features are genuine author traits, or do we need to filter them manually? Can it be done for broad linguistic domains, or do we need to search for reliable traits in each application case separately? Can we expect to find enough such features to distinguish between large numbers of authors?

\subsection{Conclusion}

We have seen that the tests traditionally used to determine the accuracy of author identification methods fail to take into account the effects of topic, style, genre etc. This has led to an overestimation of how feasible author identification is in general. Our experiments give an approximation of a lower bound for that discrepancy, but it is not possible to say if the effects are actually even bigger. This calls into question under which conditions automatic author identification is at all a feasible problem, and shows the need for methods that are explicitly designed to avoid the pitfall of topic dependence.

\section{References}

Johanna Björklund and Niklas Zechner. 2017. Syntactic methods for topic-independent authorship attribution. Natural Language Engineering, 23(5):789806.

Kim Luyckx and Walter Daelemans. 2005. Shallow text analysis and machine learning for authorship attribution. LOT Occasional Series, 4:149-160.

Rohith Menon and Yejin Choi. 2011. Domain independent authorship attribution without domain adaptation. In Proceedings of the International Conference Recent Advances in Natural Language Processing 2011, pages 309-315.

George K Mikros and Eleni K Argiri. 2007. Investigating topic influence in authorship attribution. In $P A N$.

Frederick Mosteller and David L Wallace. 1964. Inference and disputed authorship: The Federalist. Addison-Wesley.

Niklas Zechner. 2014. Effects of division of data in author identification. In Proceedings of the fifth Swedish language technology conference.

Niklas Zechner. 2017. A novel approach to text classification. Ph.D. thesis, Umeå universitet. 